\title{
Factors impacting cumulative dissipated energy levels and postoperative visual acuity outcome in cataract surgery
}

Anh D. Buil ${ }^{1+}$, Zhimin Sun ${ }^{2 \dagger}$, Yunzhen Wang ${ }^{2 \dagger}$, Shengsong Huang ${ }^{2}$, Michael Ryan ${ }^{1}$, Yinxi Yu ${ }^{3}$, Gui-Shuang Ying ${ }^{3}$, Saras Ramanathan ${ }^{1}$, Kuldev Singh ${ }^{4}$, Yangfan Yang ${ }^{2^{*}}$ and Ying Han ${ }^{1 *}$

\begin{abstract}
Purpose: To determine factors impacting cumulative dissipated energy (CDE) and postoperative best-corrected visual acuity (BCVA) in phacoemulsification.

Design: Review of 1102 cases at University of California, San Francisco (UCSF) and at Zhongshan Ophthalmic Center (ZOC), China.

Subjects: Patients who underwent cataract surgery at UCSF 03/2014-03/2019 and at ZOC 10/2018-05/2019.

Methods: Patient demographics, medical history, routine ocular examination, and surgical information, including disassembly method, complications, and surgeon training level were recorded. Univariable and multivariable regression models were used to determine factors associated with CDE and good postoperative BCVA (20/40 or better) at 1 month.

Outcome measures: CDE, postoperative BCVA.

Results: In multivariable analysis, patient age at time of surgery, diabetes, degree of nuclear sclerosis (NS), whiteto-white corneal diameter, disassembly method, preoperative BCVA, surgeon training level, and surgical center were significantly associated with CDE. $\log _{10} \mathrm{CDE}$ increased by $0.20-0.31$ for patient age $\geq 70$ years, by 0.07 if the patient had diabetes, by $0.12-0.41$ for NS grade $\geq 2$, by 0.48 per $10 \mathrm{~mm}$ increase in white-to-white corneal diameter, by 0.34-0.47 for disassembly method other than non-stop chop, by 0.16 per unit increase in preoperative logMAR BCVA, and by $>0.09$ when phacoemulsification was performed by residents early in their training. $\log _{10}$ CDE was 0.33 higher at UCSF than ZOC. In multivariable analysis, worse baseline visual acuity and age above 90 years at time of surgery decreased the odds of good $B C V A(O R=0.26$ per unit increase in preoperative logMAR $B C V A ; O R=0.12$ for age $>90)$; comorbid retinal issues decreased the odds of good postoperative $B C V A(O R=0.13-0.39)$; greater anterior chamber depth (ACD) or shorter axial length (AL), increased the odds of good postoperative outcome $(O R=2.64$ per $1 \mathrm{~mm}$ increase $A C D, O R=0.84$ per $1 \mathrm{~mm}$ increase $\mathrm{AL}$ ).
\end{abstract}

*Correspondence: yangyangfan@gzzoc.com; Ying.Han@ucsf.edu

${ }^{\dagger}$ Anh D. Bui, Zhimin Sun and Yunzhen Wang contributed equally to this

work.

1 Department of Ophthalmology, University of California, San Francisco,

490 Illinois Street, San Francisco, CA 94158, USA

${ }^{2}$ State Key Laboratory of Ophthalmology, Zhongshan Ophthalmic Center,

Sun Yat-sen University, Guangzhou, China

Full list of author information is available at the end of the article

(c) The Author(s) 2021. Open Access This article is licensed under a Creative Commons Attribution 4.0 International License, which permits use, sharing, adaptation, distribution and reproduction in any medium or format, as long as you give appropriate credit to the original author(s) and the source, provide a link to the Creative Commons licence, and indicate if changes were made. The images or other third party material in this article are included in the article's Creative Commons licence, unless indicated otherwise in a credit line to the material. If material is not included in the article's Creative Commons licence and your intended use is not permitted by statutory regulation or exceeds the permitted use, you will need to obtain permission directly from the copyright holder. To view a copy of this licence, visit http://creativecommons.org/licenses/by/4.0/. The Creative Commons Public Domain Dedication waiver (http://creativeco mmons.org/publicdomain/zero/1.0/) applies to the data made available in this article, unless otherwise stated in a credit line to the data. 
Conclusions: Cataract grade determined by slit lamp exam and, for the first time, older patient age, were noted to be important predictors of high CDE. CDE was not a risk factor for postoperative BCVA measured at postoperative 1 month. When surgery was performed by trainees under supervision, lower training level was associated with higher $C D E$, but not with worse postoperative BCVA.

Keywords: Cataract, Phacoemulsification, Cumulative dissipated energy, CDE, Visual outcome

Cataract surgery is the most commonly performed ophthalmic surgery. Phacoemulsification has become the method of choice for lens removal in uncomplicated cataracts. The outcomes of phacoemulsification surgery for vision restoration are excellent [1]. The present goals for improvements in phacoemulsification methods are thus focused on optimizing refractive results, accelerating recovery speed, and reducing complication rates.

Two key objectives during phacoemulsification are to: 1) avoid or minimize any trauma to nearby ocular issue and 2) maximize the visual acuity after phacoemulsification. Besides direct instrumental damage to ocular tissues, the degree of ultrasonic exposure during phacoemulsification is a hidden but important determinant of iatrogenic injury, such as corneal endothelial cell loss, which may lead to long-term complications. Ultrasonic exposure results in heat dissipation within the corneal tissues and may cause acoustic cavitation and hydroxyl radical generation within the anterior chamber $[2,3]$. While decline of endothelial cell count is expected with advancing age, accelerated rates of endothelial cell loss have been reported after surgery, ranging from 4-20\% within the year following cataract surgery [4-7]. The amount of ultrasonic exposure during phacoemulsification is typically quantified using cumulative dissipated energy (CDE), which is measured and reported by the phacoemulsification systems. The second outcome is visual acuity after phacoemulsification, which is the primary goal of cataract surgery in majority of cases.

Despite the fact that several studies looked at factors predictive for high CDE levels and final visual acuity $[6$, 8], our understanding of the patient-specific and surgery-related factors that contribute to the variation of intraoperative $C D E$ and final visual acuity remains limited, especially for trainees' performed phacoemulsification. Limitations from previous studies include that cohort sizes were typically small and some commonly encountered factors (such as age at time of surgery) were not analyzed, potentially limiting the generalizability of the study findings $[6,8]$. Furthermore, some factors that have been analyzed are not always easily obtainable clinically. In particular, while it has been well-established that lens density affects CDE, this association has mainly been studied by slit-lamp images with standard photographic charts of the Lens
Opacities Classification System III (LOCS III) system or a Scheimpflug imaging system (Pentacam), neither system is commonly used in daily practice. The relationship between CDE and clinical grading of lens density during slit lamp examination has not been studied. Finally, the association between CDE and postoperative outcomes is also limited.

The purpose of this study is to investigate a broad set of risk factors that affect CDE levels and postoperative BCVA in a large cohort of patients who had phacoemulsification surgery at two large and independent teaching centers. Identification of factors associated with $\mathrm{CDE}$ and visual acuity may improve teaching of phacoemulsification surgery, guide surgeons in optimizing phacoemulsification and better prepare for difficult cases to improve surgical outcomes.

\section{Methods}

\section{Study population}

This study was conducted in compliance with the rules and regulations of the Health Insurance Portability and Accountability Act and all applicable federal and state laws, and in adherence to the tenets of the Declaration of Helsinki. The study was approved by the Institutional Review Board at the University of California, San Francisco (UCSF) and Zhongshan Ophthalmic Center (ZOC) in Guangzhou, China.

This study included patients who met the inclusion criteria and underwent phacoemulsification and intraocular lens implantation at UCSF between March 1, 2014 and March 1, 2019. Data from patients who underwent phacoemulsification and intraocular lens implantation at ZOC from October 1, 2018 to May 1, 2019 was accumulated. The inclusion criteria included: (1) ability to identify primary surgeon, (2) adult patients (age $>18$ years). The exclusion criteria included: (1) missing CDE data, (2) combined cases, such as combined corneal transplants, glaucoma surgeries, or retinal surgeries. Corneal or retinal conditions, glaucoma, or uveitis were not criteria for exclusion and were included as risk factors in the analysis. Demographic and clinical information was recorded, including age, gender, race, medical and surgical history, medications, allergies, and family history of ocular disease. 


\section{Cataract grading}

Cataract grading was performed by the surgeon on clinical slit lamp examination during the preoperative evaluation. A modified Lens Opacities Classification System III (LOCS III) was used to grade the cataracts in the ophthalmology clinic at both UCSF and ZOC [9]. Observers were attending physicians who were fully trained on the classification system. For nuclear cataract, the range was from grade 1 for mild nuclear opacification and color to grade 4 for severe nuclear opacification. Cortical cataract was graded by degree of intrapupillary space obscured, with less than $10 \%$ for grade $1,10-50 \%$ for grade 2, $50-90 \%$ for grade 3 , and more than $90 \%$ for grade 4 . Posterior subcapsular cataract was graded by degree of posterior capsule obscured, with less than $3 \%$ for grade 1 , $3-30 \%$ for grade $2,30-50 \%$ for grade 3 , and more than $50 \%$ for grade 4 .

\section{Cataract surgical procedure}

Phacoemulsification was performed in standard fashion under topical or sub-Tenon's anesthesia (Centurion; Alcon Laboratories, Inc). Briefly, after a temporal clear corneal incision was created, an anterior capsulorhexis was performed, followed by hydrodissection with or without hydrodelineation. The lens nucleus was removed utilizing non-stop chop, stop and chop, or divide and conquer. Both centers used the same strategy to optimize the phacoemulsification machine setting parameters. An acrylic intraocular lens (IOL) was inserted into the bag after removing the epinucleus and cortex when no complications occurred. Otherwise, the IOL was placed into the sulcus or anterior chamber or the patient was left aphakic, and complications (e.g., posterior capsule rupture with or without vitrectomy, dropped lens, and zonular dialysis) were noted for inclusion in the analysis as a risk factor. Prophylactic intracameral or subconjunctival antibiotic was given.

\section{Outcome measures and analyzed factors}

$\mathrm{CDE}$, recorded from the phacoemulsification system, was analyzed as a continuous response variable. Preoperative and postoperative Snellen best-corrected visual acuity (BCVA) were measured on the logarithm of the minimum angle of resolution (logMAR) scale. Postoperative BCVA was transformed into a dichotomous outcome, with $\operatorname{logMAR} \leq 0.3$ (Snellen equivalent 20/40) considered as good postoperative BCVA and logMAR $>0.3$ considered as poor postoperative BCVA. Postoperative BCVA was measured with manifest refraction approximately 1 month after cataract surgery. Twenty-one risk factors (Table 1) were recorded and analyzed for association with these response variables (e.g., CDE and BCVA).
These risk factors included cataract grade (see "Cataract Grading"), preoperative BCVA and postoperative BCVA, and other factors grouped according to the following categories: patient demographics, patient ocular geometry, patient medical history, surgical method, and surgeon training and affiliation. Complications (e.g., capsular rupture with or without vitrectomy, dropped lens, zonular dialysis) were also documented.

\section{Statistical analysis}

Continuous factors were summarized using mean and standard deviation, categorical and factors were summarized using percentage. Due to the right skewed distribution of CDE, CDE was first transformed as $\log 10$ $(\mathrm{CDE}+1)$ to normalize its distribution, and this logtransformed measure was used for statistical analysis. Univariable and multivariable linear regression models were used to identify factors associated with CDE. Univariable and multivariable logistic regression models were used to identify factors associated with good postoperative BCVA (i.e., logMAR $\leq 0.3$ ) at 1 month. In the multivariable analyses for CDE and BCVA, the initial multivariable regression model included all the factors with $p \leq 0.20$ from the univariable analysis, the model then went through the backward variable selection by keeping the factors with $p \leq 0.05$ in the final multivariate model. All statistical analyses were performed using the Statistical Analysis Software (SAS version 9.4, North Carolina, USA).

\section{Results \\ Demographics}

There were 1294 cases screened for this study. After excluding 64 cases due to missing $\mathrm{CDE}$ and 128 cases due to cataract surgery combined with other ocular surgical procedures, a total of 1102 cases (864 cases from UCSF and 238 cases from ZOC) with phacoemulsification were included into analysis. Table 1 summarizes the demographics and clinical characteristics of the study subjects. The mean age ( \pm standard deviation) of patients was $70.7 \pm 10.6$ years, and $39.6 \%$ were male. Regarding medical and ocular history, $23.4 \%$ were diabetic, $0.7 \%$ had comorbid uveitis, $0.6 \%$ had corneal scars or transplants, $24.4 \%$ had glaucoma, and 5.8\% had retinal pathologies or history of prior retinal procedures. In terms of patient ocular geometry parameters, anterior chamber depth was $3.1 \pm 0.5 \mathrm{~mm}$, and axial length was $24.1 \pm 1.7 \mathrm{~mm}$. Regarding cataract grading, the majority of them were clinical nuclear sclerotic (NS) cataract grade $2(47.2 \%)$, the average clinical cortical cataract grade was 0.86 , and the average posterior subcapsular cataract grade was 0.64 . Complications during surgery included $0.5 \%$ cases with capsular 
Table 1 Baseline characteristics of study participants in UCSF cohort and ZOC cohort

\begin{tabular}{|c|c|c|c|}
\hline & UCSF ( $N=864$ eyes $)$ & $\mathrm{ZOC}(\mathrm{N}=\mathbf{2 3 8}$ eyes $)$ & $\begin{array}{l}\text { Combined } \\
\text { ( } N=1102 \text { eyes) }\end{array}$ \\
\hline \multicolumn{4}{|l|}{ Patient-Level Characteristics } \\
\hline \multicolumn{4}{|l|}{ Age at the time of surgery (years) } \\
\hline Mean (SD) & $72.1(9.8)$ & $65.7(11.8)$ & $70.7(10.6)$ \\
\hline$<60$ & $82(9.5 \%)$ & $55(23.1 \%)$ & $137(12.4 \%)$ \\
\hline $60-69$ & $258(29.9 \%)$ & $91(38.2 \%)$ & $349(31.7 \%)$ \\
\hline $70-79$ & $354(41.0 \%)$ & $70(29.4 \%)$ & $424(38.5 \%)$ \\
\hline $80-89$ & $141(16.3 \%)$ & $22(9.2 \%)$ & $163(14.8 \%)$ \\
\hline$>=90$ & $29(3.4 \%)$ & $0(0.0 \%)$ & $29(2.6 \%)$ \\
\hline \multicolumn{4}{|l|}{ Gender } \\
\hline Male & $327(37.8 \%)$ & $109(45.8 \%)$ & $436(39.6 \%)$ \\
\hline Female & $537(62.2 \%)$ & $129(54.2 \%)$ & $666(60.4 \%)$ \\
\hline Diabetics: Yes (\%) & $213(24.7 \%)$ & $45(18.9 \%)$ & $258(23.4 \%)$ \\
\hline \multicolumn{4}{|l|}{ Eye-Level Characteristics } \\
\hline Uveitis comorbid: Yes (\%) & $6(0.7 \%)$ & $2(0.8 \%)$ & $8(0.7 \%)$ \\
\hline \multicolumn{4}{|l|}{ Cornea comorbid } \\
\hline No & $862(99.8 \%)$ & $234(98.3 \%)$ & 1096 (99.5\%) \\
\hline Scarred or cloudy cornea & $1(0.1 \%)$ & $4(1.7 \%)$ & $5(0.5 \%)$ \\
\hline Penetrating keratoplasty/endothelial keratoplasty & $1(0.1 \%)$ & $0(0.0 \%)$ & $1(0.1 \%)$ \\
\hline \multicolumn{4}{|l|}{ Glaucoma comorbid } \\
\hline No & $622(72.0 \%)$ & $212(89.1 \%)$ & $834(75.7 \%)$ \\
\hline Incisional surgery & $12(1.4 \%)$ & $12(5.0 \%)$ & $24(2.2 \%)$ \\
\hline Pseudoexfoliation glaucoma & $24(2.8 \%)$ & $0(0.0 \%)$ & $24(2.2 \%)$ \\
\hline Primary angle closure disease & $43(5.0 \%)$ & $14(5.9 \%)$ & $57(5.2 \%)$ \\
\hline Other & $163(18.9 \%)$ & $0(0.0 \%)$ & $163(14.8 \%)$ \\
\hline \multicolumn{4}{|l|}{ Retina comorbid } \\
\hline None & $824(95.4 \%)$ & $215(90.3 \%)$ & $1039(94.3 \%)$ \\
\hline Pars plana vitrectomy & $4(0.5 \%)$ & $23(9.7 \%)$ & $27(2.5 \%)$ \\
\hline Other & $36(4.2 \%)$ & $0(0.0 \%)$ & $36(3.3 \%)$ \\
\hline \multicolumn{4}{|l|}{ Nuclear sclerotic cataract grade } \\
\hline Unknown & $6(0.7 \%)$ & $0(0.0 \%)$ & $6(0.5 \%)$ \\
\hline 0 & $23(2.7 \%)$ & $0(0.0 \%)$ & $23(2.1 \%)$ \\
\hline 1 & $160(18.5 \%)$ & $17(7.1 \%)$ & $177(16.1 \%)$ \\
\hline 2 & $471(54.5 \%)$ & $49(20.6 \%)$ & $520(47.2 \%)$ \\
\hline 3 & $192(22.2 \%)$ & $139(58.4 \%)$ & $331(30.0 \%)$ \\
\hline 4 & $12(1.4 \%)$ & $33(13.9 \%)$ & $45(4.1 \%)$ \\
\hline \multicolumn{4}{|l|}{ Cortical cataract grade } \\
\hline Missing & $3(0.3 \%)$ & $0(0.0 \%)$ & $3(0.3 \%)$ \\
\hline 0 & $565(65.4 \%)$ & $13(5.5 \%)$ & $578(52.5 \%)$ \\
\hline 1 & $91(10.5 \%)$ & $115(48.3 \%)$ & $206(18.7 \%)$ \\
\hline 2 & $145(16.8 \%)$ & $63(26.5 \%)$ & $208(18.9 \%)$ \\
\hline 3 & $54(6.3 \%)$ & $45(18.9 \%)$ & $99(9.0 \%)$ \\
\hline 4 & $6(0.7 \%)$ & $2(0.8 \%)$ & $8(0.7 \%)$ \\
\hline \multicolumn{4}{|l|}{ Posterior subcapsular cataract grade } \\
\hline Missing & $4(0.5 \%)$ & $0(0.0 \%)$ & $4(0.4 \%)$ \\
\hline 0 & 721 (83.4\%) & $0(0.0 \%)$ & 721 (65.4\%) \\
\hline 1 & 51 (5.9\%) & 140 (58.8\%) & $191(17.3 \%)$ \\
\hline 2 & 37 (4.3\%) & 45 (18.9\%) & $82(7.4 \%)$ \\
\hline 3 & 42 (4.9\%) & 28 (11.8\%) & 70 (6.4\%) \\
\hline 4 & $9(1.0 \%)$ & 25 (10.5\%) & 34 (3.1\%) \\
\hline
\end{tabular}


Table 1 (continued)

\begin{tabular}{|c|c|c|c|}
\hline & UCSF ( $N=864$ eyes) & $\mathrm{ZOC}(N=238$ eyes $)$ & $\begin{array}{l}\text { Combined } \\
(N=1102 \text { eyes })\end{array}$ \\
\hline \multicolumn{4}{|l|}{ Axial length (mm) } \\
\hline N & 859 & 235 & 1094 \\
\hline Mean (SD) & $24.1(1.6)$ & $24.3(2.2)$ & $24.1(1.7)$ \\
\hline \multicolumn{4}{|l|}{ Anterior chamber depth $(\mathrm{mm})$} \\
\hline N & 845 & 235 & 1080 \\
\hline Mean (SD) & $3.1(0.5)$ & $3.1(0.5)$ & $3.1(0.5)$ \\
\hline \multicolumn{4}{|l|}{ White-to-white corneal diameter (mm) } \\
\hline N & 858 & 234 & 1092 \\
\hline Mean (SD) & $11.8(0.5)$ & $11.7(0.7)$ & $11.7(0.6)$ \\
\hline \multicolumn{4}{|l|}{ Pre-surgery logMAR visual acuity } \\
\hline $\mathrm{N}$ & 864 & 238 & 1102 \\
\hline Mean (SD) & $0.5(0.4)$ & $1.1(0.5)$ & $0.6(0.5)$ \\
\hline \multicolumn{4}{|l|}{ Surgery-Level Characteristics } \\
\hline \multicolumn{4}{|l|}{ Surgeon training level } \\
\hline Second year resident & $2(0.2 \%)$ & $0(0.0 \%)$ & $2(0.2 \%)$ \\
\hline Early third year resident & $192(22.2 \%)$ & $0(0.0 \%)$ & $192(17.4 \%)$ \\
\hline Late third year resident & $236(27.3 \%)$ & $0(0.0 \%)$ & $236(21.4 \%)$ \\
\hline Fellow & $246(28.5 \%)$ & $0(0.0 \%)$ & $246(22.3 \%)$ \\
\hline Attending & $188(21.8 \%)$ & $238(100.0 \%)$ & $426(38.7 \%)$ \\
\hline \multicolumn{4}{|l|}{ Disassembly method } \\
\hline Unknown & $1(0.1 \%)$ & $0(0.0 \%)$ & $1(0.1 \%)$ \\
\hline Divide and conquer & $20(2.3 \%)$ & $6(2.5 \%)$ & $26(2.4 \%)$ \\
\hline Stop and chop & $135(15.6 \%)$ & $232(97.5 \%)$ & $367(33.3 \%)$ \\
\hline Non-stop chop & $705(81.6 \%)$ & $0(0.0 \%)$ & $705(64.0 \%)$ \\
\hline 2 handed technique & $2(0.2 \%)$ & $0(0.0 \%)$ & $2(0.2 \%)$ \\
\hline Using the epinuclear setting & $1(0.1 \%)$ & $0(0.0 \%)$ & $1(0.1 \%)$ \\
\hline \multicolumn{4}{|c|}{ Pupil expansion or capsular support devices } \\
\hline None & $835(96.6 \%)$ & $236(99.2 \%)$ & $1071(97.2 \%)$ \\
\hline Iris hooks & $4(0.5 \%)$ & $1(0.4 \%)$ & $5(0.5 \%)$ \\
\hline Mackool hooks & $1(0.1 \%)$ & $0(0.0 \%)$ & $1(0.1 \%)$ \\
\hline Capsular tension ring & $2(0.2 \%)$ & $1(0.4 \%)$ & $3(0.3 \%)$ \\
\hline Mackool + Capsular tension ring & $1(0.1 \%)$ & $0(0.0 \%)$ & $1(0.1 \%)$ \\
\hline Malyugen ring & $20(2.3 \%)$ & $0(0.0 \%)$ & $20(1.8 \%)$ \\
\hline I-Ring & $1(0.1 \%)$ & $0(0.0 \%)$ & $1(0.1 \%)$ \\
\hline Loose zonules & $6(0.7 \%)$ & $6(2.5 \%)$ & $12(1.1 \%)$ \\
\hline \multicolumn{4}{|l|}{ Complication status } \\
\hline None & $845(97.8 \%)$ & $236(99.2 \%)$ & 1081 (98.1\%) \\
\hline Capsular rupture without vitrectomy & $5(0.6 \%)$ & $0(0.0 \%)$ & $5(0.5 \%)$ \\
\hline Capsular rupture with vitrectomy & $10(1.2 \%)$ & $0(0.0 \%)$ & $10(0.9 \%)$ \\
\hline Dropped lens & $0(0.0 \%)$ & $2(0.8 \%)$ & $2(0.2 \%)$ \\
\hline Zonular dialysis, vitrectomy & $1(0.1 \%)$ & $0(0.0 \%)$ & $1(0.1 \%)$ \\
\hline Other & $3(0.3 \%)$ & $0(0.0 \%)$ & $3(0.3 \%)$ \\
\hline \multicolumn{4}{|l|}{ CDE (Percent-seconds) } \\
\hline N & 864 & 234 & 1098 \\
\hline Mean (SD) & $9.4(13.0)$ & $11.1(9.9)$ & $9.8(12.5)$ \\
\hline Median & 5.3 & 8.3 & 6.0 \\
\hline Q1, Q3 & $2.6,10.6$ & $4.5,13.3$ & $2.9,11.5$ \\
\hline Range & $(0.0-119.0)$ & $(0.2-55.3)$ & $(0.0-119.0)$ \\
\hline
\end{tabular}


Table 1 (continued)

\begin{tabular}{|c|c|c|c|}
\hline & UCSF ( $N=864$ eyes) & $\mathrm{ZOC}(\mathrm{N}=238$ eyes $)$ & $\begin{array}{l}\text { Combined } \\
\text { ( } N=1102 \text { eyes) }\end{array}$ \\
\hline \multicolumn{4}{|c|}{$\log 10(C D E+1)$} \\
\hline N & 864 & 234 & 1098 \\
\hline Mean (SD) & $0.8(0.4)$ & $1.0(0.3)$ & $0.9(0.4)$ \\
\hline Median & 0.8 & 1.0 & 0.8 \\
\hline Q1, Q3 & $0.6,1.1$ & $0.7,1.2$ & $0.6,1.1$ \\
\hline Range & $(0.0-2.1)$ & $(0.1-1.8)$ & $(0.0-2.1)$ \\
\hline
\end{tabular}

Abbreviations: logMAR Logarithm of the minimum angle of resolution

rupture without vitrectomy, $0.9 \%$ cases had capsular rupture with vitrectomy, $0.1 \%$ cases with zonular dialysis requiring vitrectomy, and $0.3 \%$ cases with other unspecified complications. Attendings or trainees performed the surgeries at UCSF, while attendings performed all surgeries at ZOC. The trainees at UCSF included 2nd year residents, early 3rd year residents who were within the first 6 months of their 3rd year, late 3 rd year residents who were in the second 6 months of their 3rd year, and fellows trained for either glaucoma or cornea. All trainee-performed phacoemulsifications were under direct supervision of attending surgeons.

\section{Factors associated with cumulative dissipated energy}

The results from the univariable analysis are reported in Supp. Table 1. In the multivariable analysis, patient age at time of surgery, diabetes, white-to-white corneal diameter, clinical NS grade, preoperative BCVA, cataract disassembly method, surgeon training level, and surgical center were significantly associated with $\mathrm{CDE}$ levels (Table 2). Older age and diabetes were associated with higher $\mathrm{CDE}$ (increase in $\log _{10} \mathrm{CDE}$ by $0.20-0.31$ for age $\geq 70$ years, $p<0.001$; increase in $\log _{10} \mathrm{CDE}$ by 0.07 with diabetes, $p=0.007$ ); higher NS grade and greater white-to-white corneal diameter were significantly associated with higher $\mathrm{CDE}$ (increase in $\log _{10} \mathrm{CDE}$ by 0.12 0.41 for NS grade $\geq 2, p<0.001$; increase in $\log _{10} \mathrm{CDE}$ by 0.48 per $10 \mathrm{~mm}$ increase in white-to-white corneal diameter, $p=0.008$ ); worse preoperative BCVA was associated with higher $\mathrm{CDE}$ (increase in $\log _{10} \mathrm{CDE}=0.16$ per $\log$ MAR unit increase, $p<0.001$ ); use of a disassembly method other than non-stop chop was associated with higher CDE with an increase in $\log _{10} \mathrm{CDE}$ of 0.34 for stop and chop method and 0.47 for other methods. CDE was also higher at UCSF than ZOC by $0.33 \log 10 \mathrm{CDE}$ $(p<0.001)$. CDE levels were significantly lower when surgery was performed by attendings, fellows, or third year residents compared to second year residents (decrease in $\log _{10}$ CDE by $>0.09, p=0.03$ ).

\section{Factors associated with postoperative visual acuity outcome}

Results from the univariable analysis for factors associated with good VA outcome (20/40 or better) were shown in (Suppl. Table 2). In the multivariable analysis, age above 90 years old, retinal disease, axial length $(\mathrm{AL})$, anterior chamber depth $(\mathrm{ACD})$, and preoperative BCVA were significantly associated with postoperative visual acuity outcome (Table 3 ). Patients older than 90 years had lower odds of good postoperative BCVA compared to patients younger than 60 years $(\mathrm{OR}=0.12$, $p<0.001)$. Patients with comorbid retinal issues had lower odds of good vision $(\mathrm{OR}=0.39, p<0.01)$. Greater $\mathrm{AL}$ was associated with lower odds of good postoperative $\mathrm{BCVA}(\mathrm{OR}=0.84$ per $1 \mathrm{~mm}$ increase $\mathrm{AL})$; greater ACD increased the odds of good postoperative outcome $(\mathrm{OR}=2.64$ per $1 \mathrm{~mm}$ increase $\mathrm{ACD})$; worse baseline visual acuity was associated with lower odds of good postoperative $B C V A(O R=0.26$ per unit increase in preoperative BCVA logMAR, $p<0.001)$. Visual acuity outcome was not significantly different between the two surgical centers. Notably, NS grade, cataract disassembly method, surgeon training level, and CDE level were not significantly associated with good visual acuity outcome.

\section{Conclusion and discussion}

In this study of over 1000 cases of phacoemulsification at two independent surgical centers, we tested a broad set of factors for association with intraoperative CDE levels and postoperative BCVA. The motivation for our study is to provide surgeons with reliable risk factor charts including factors that can be practically obtained clinically, to better predict the levels of ultrasound emitted during phacoemulsification, and to better predict the visual acuity outcome when cases are performed with or without trainees. This study provides important information to determine which cataract cases are appropriate for trainees to guide teaching phacoemulsification to residents.

Although the impact of ultrasonic exposure in causing iatrogenic injury of ocular tissue remain debated, current consensus among cataract surgeons is to limit ultrasonic 
Table 2 Multivariable Analysis of Factors Associated with Cumulative Dissipated Energy

\begin{tabular}{|c|c|c|c|c|c|c|}
\hline \multirow[t]{2}{*}{ Characteristics } & & \multicolumn{5}{|c|}{ Combined ( $N=1082$ eyes) } \\
\hline & & $\mathrm{N}$ of eyes & $\begin{array}{l}\text { Log10 (CDE+ 1) } \\
\text { Adjusted Mean (SE) }\end{array}$ & Adjusted Difference $(95 \% \mathrm{Cl})$ & $P$-value & Overall P-value \\
\hline \multicolumn{7}{|l|}{ Patient-Level Characteristics } \\
\hline \multirow[t]{5}{*}{ Age at the time of surgery } & $<60$ & 132 & $0.70(0.03)$ & REF & & $<0.001$ \\
\hline & $60-69$ & 340 & $0.78(0.02)$ & $0.08(0.01,0.16)$ & 0.03 & \\
\hline & $70-79$ & 420 & $0.90(0.02)$ & $0.20(0.12,0.27)$ & $<0.001$ & \\
\hline & $80-89$ & 162 & $0.99(0.03)$ & $0.29(0.20,0.38)$ & $<0.001$ & \\
\hline & $>=90$ & 28 & $1.01(0.08)$ & $0.31(0.13,0.48)$ & $<0.001$ & \\
\hline \multirow[t]{2}{*}{ Diabetics } & No & 828 & $0.84(0.01)$ & REF & & 0.007 \\
\hline & Yes & 254 & $0.91(0.02)$ & $0.07(0.02,0.12)$ & 0.007 & \\
\hline \multicolumn{7}{|l|}{ Eye-Level Characteristics } \\
\hline \multirow[t]{5}{*}{ Nuclear sclerotic cataract grade } & 0 & 21 & $0.68(0.06)$ & REF & & $<0.001$ \\
\hline & 1 & 173 & $0.72(0.03)$ & $0.04(-0.08,0.16)$ & 0.56 & \\
\hline & 2 & 516 & $0.81(0.02)$ & $0.12(0.00,0.24)$ & 0.046 & \\
\hline & 3 & 327 & $0.97(0.02)$ & $0.29(0.17,0.41)$ & $<0.001$ & \\
\hline & 4 & 45 & $1.10(0.05)$ & $0.41(0.26,0.57)$ & $<0.001$ & \\
\hline White-to-white corneal diameter & Every $10 \mathrm{~mm}$ increase & 1082 & & $0.48(0.13,0.83)$ & & 0.008 \\
\hline Pre-surgery logMAR visual acuity & Every 1 unit increase & 1082 & & $0.16(0.11,0.22)$ & & $<0.001$ \\
\hline \multicolumn{7}{|l|}{ Surgery-Level Characteristics } \\
\hline \multirow[t]{5}{*}{ Surgeon training level } & Second year resident & 2 & $0.97(0.06)$ & REF & & 0.03 \\
\hline & Early third year resident & 190 & $0.85(0.02)$ & $-0.12(-0.24,-0.00)$ & 0.04 & \\
\hline & Late third year resident & 234 & $0.82(0.02)$ & $-0.16(-0.27,-0.04)$ & 0.006 & \\
\hline & Fellow & 242 & $0.85(0.03)$ & $-0.13(-0.25,-0.00)$ & 0.04 & \\
\hline & Attending & 414 & $0.88(0.02)$ & $-0.09(-0.21,0.02)$ & 0.02 & \\
\hline \multirow[t]{3}{*}{ Disassembly method } & Stop and chop & 360 & $1.07(0.03)$ & REF & & $<0.001$ \\
\hline & Non-stop chop & 693 & $0.73(0.02)$ & $-0.34(-0.41,-0.27)$ & $<0.001$ & \\
\hline & Other & 29 & $1.19(0.06)$ & $0.13(0.00,0.26)$ & 0.048 & \\
\hline \multirow[t]{2}{*}{ Center } & UCSF & 851 & $0.93(0.02)$ & REF & & $<0.001$ \\
\hline & $\mathrm{ZOC}$ & 231 & $0.59(0.04)$ & $-0.33(-0.43,-0.24)$ & $<0.001$ & \\
\hline
\end{tabular}

Table 3 Multivariable Analysis of Factors Associated with Post-surgery Good Vision (VA $\leq 0.3$ logMAR)

\begin{tabular}{|c|c|c|c|c|c|c|}
\hline \multirow[t]{2}{*}{ Characteristics } & & \multicolumn{5}{|c|}{ Combined ( $N=1079$ eyes) } \\
\hline & & $\mathrm{N}$ of eyes & $\mathrm{n}(\%)$ of good vision & $\begin{array}{l}\text { Adjusted Odds } \\
\text { ratioa }(95 \% \mathrm{Cl})\end{array}$ & $P$-value & Overall P-value \\
\hline \multirow[t]{5}{*}{ Age at the time of surgery } & $<60$ & 132 & $101(76.5 \%)$ & REF & & 0.002 \\
\hline & $60-69$ & 340 & $280(82.4 \%)$ & $1.05(0.53,2.08)$ & 0.90 & \\
\hline & $70-79$ & 418 & $333(79.7 \%)$ & $0.72(0.36,1.43)$ & 0.34 & \\
\hline & $80-89$ & 161 & $124(77.0 \%)$ & $0.52(0.24,1.13)$ & 0.10 & \\
\hline & $>=90$ & 28 & $7(25.0 \%)$ & $0.12(0.03,0.43)$ & $<0.001$ & \\
\hline \multirow[t]{3}{*}{ Retina comorbid } & None & 1016 & $822(80.9 \%)$ & REF & & $<0.001$ \\
\hline & Pars plana vitrectomy & 27 & $12(44.4 \%)$ & $0.39(0.18,0.88)$ & 0.001 & \\
\hline & Other & 36 & $11(30.6 \%)$ & $0.13(0.05,0.33)$ & 0.02 & \\
\hline Axial length & Every $1 \mathrm{~mm}$ increase & 1079 & $845(78.3 \%)$ & $0.84(0.76,0.93)$ & & $<0.001$ \\
\hline Anterior chamber depth & Every $1 \mathrm{~mm}$ increase & 1079 & $845(78.3 \%)$ & $2.64(1.75,3.97)$ & & $<0.001$ \\
\hline Pre-surgery logMAR visual acuity & Every 1 unit increase & 1079 & 845 (78.3\%) & $0.26(0.19,0.35)$ & & $<0.001$ \\
\hline
\end{tabular}

${ }^{a}$ Odds ratio was defined as the odds of good post-surgery visual outcome with logMAR BCVA score $\leq 0.3$ 
exposure to the ocular tissue during the surgery. Some studies have shown positive correlation between $\mathrm{CDE}$ levels and the rate of endothelial cell loss, while others have shown no significant correlation $[6-8,10,11]$. The long-term effect of $\mathrm{CDE}$ on cornea condition needs to be further studied. We postulated that while CDE level may not be relevant in determining outcomes of routine cases, it may be important for patients at higher risk for complications. Predicting CDE before cataract surgery for older patients or patients with history of ocular disease might help guide the best course of action to protect corneal tissue.

We found that patient age, clinical NS grade, cataract disassembly method, white-to-white corneal diameter, preoperative $\mathrm{BCVA}$, surgeon training level and surgery center are independent predictors of CDE levels. Comparing within similar cataract gradings, patients of age 70 or older required greater phacoemulsification energy than younger patients, which, to our knowledge, has not been previously reported. This association is clinically important and should be considered when deciding optimal timing for cataract surgery, particularly in eyes with ocular co-morbidities such as corneal disease. We also found that CDE levels were positively correlated with clinical NS grade, which is consistent with the previous reports regarding the relationship between lens density and CDE $[12,13]$. This result is particularly noteworthy as it suggests that Scheimpflug images may not be necessary for predicting CDE values during surgery, as cataract density graded under slit lamp exam may serve as a surrogate measure. The association of the non-stop cataract disassembly method with lower CDE values agrees with prior studies [11, 14].

Not surprisingly, we found that second year residents used higher CDE levels than third year residents, fellows or attendings, which is a finding not previously reported. This is consistent with an early trainee's unfamiliarity with the phacoemulsification system and the need for trainees to develop dexterity over time. Importantly, our findings can be taken into account when deciding which phacoemulsification cases are appropriate for teaching.. For example, early trainees may consider treating younger patients and those with less dense cataracts. Our analysis revealed that $\mathrm{CDE}$ levels were different across the two centers. Inclusion of trainees at UCSF is a possible reason. In addition, the difference could be also explained by procedural differences. This effect may be investigated in future dedicated studies comparing data across multiple centers.

Regarding visual acuity outcome at 1 month postoperatively, we found that it was significantly associated with preoperative BCVA, AL, and ACD. Notably, while
NS grade, cataract disassembly method, surgeon training level and surgical center were associated with CDE levels, these factors were not associated with postoperative BCVA. CDE levels alone were also not associated with postoperative BCVA. Together, these results suggest that while CDE levels are heterogenous across cases and can be higher when surgery is performed by supervised residents in their early training stages, this variation may have no consequence on surgical outcome as quantified by postoperative BCVA, when direct supervision is provided. It should be noted that the visual acuity was measured 1 month postoperatively, and tissue damage that may occur during surgery may take months or years to manifest clinically. Future studies will be necessary to fully understand the factors determining cataract surgery outcome in the long-term.

A limitation of the study is that there was no trainee data at ZOC to compare with that at UCSF. Secondly, ZOC and UCSF have disparate patient populations. However, this may help generalize our results to different ethnicities and surgical settings. Thirdly, some phacoemulsification parameters, such as phacoemulsification time, were not included in this large cohort study. However, because CDE integrates the amount of ultrasonic energy delivered to the eye over the course of the procedure, there is a structural positive association between effective phacoemulsification time and CDE. Thus, effective phacoemulsification time is implicitly embedded in this analysis through $\mathrm{CDE}$, and our results that $\mathrm{CDE}$ is not significantly associated with postoperative BCVA are consistent with these prior studies reporting that effective phacoemulsification time is not associated with postoperative outcome [15]. Lastly, because of the low rate of complications, we were not able to analyze the association between complications and CDE postoperative visual acuity.

Overall, our series of cases from two independent institutions reveals that $\mathrm{CDE}$ levels and postoperative BCVA are affected by a variety of factors. Clinically determined NS grade under slit lamp, age of surgery, and resident at early training stage are newly identified risk factors correlated with CDE levels. BCVA at 1 month postoperatively is uncorrelated with $C D E$ levels during cataract surgery. Greater experience of the primary surgeon was associated with lower CDE levels. However, our study indicates that in terms of visual acuity outcome, phacoemulsification is as safe and effective when performed by supervised residents as when performed by attendings.

None of the authors have any conflicts of interest or financial disclosures. 


\section{Supplementary Information}

The online version contains supplementary material available at https://doi. org/10.1186/s12886-021-02205-W.

\section{Additional file 1.}

\section{Acknowledgements}

Not applicable.

\section{Authors' contributions}

Concept and design: ADB, MR, YY1, GY, SR, KS, YY2, YH. Data collection: ZS, YW, SH. Tables: ADB, YY1, GY, YH. Writing the article: ADB, YH. Final approval: all authors.

\section{Funding}

National Eye Institute (NEI EY028747-01) funding to Dr. Y. Han.

\section{Availability of data and materials}

The datasets used and/or analyzed during the current study are available from the corresponding author on reasonable request.

\section{Declarations}

\section{Ethics approval and consent to participate}

This study was conducted in compliance with the rules and regulations of the Health Insurance Portability and Accountability Act and all applicable federal and state laws, and in adherence to the tenets of the Declaration of Helsinki. The study was approved by the Institutional Review Board at the University of California, San Francisco (UCSF) and Zhongshan Ophthalmic Center (ZOC) in Guangzhou, China.

\section{Consent for publication}

The need for consent was waived by the Institutional Review Board at the University of California, San Francisco (No. 19-28289) and Zhongshan Ophthalmic Center in Guangzhou, China given the retrospective nature of the study.

\section{Competing interests}

There are no conflicts of interest to declare for all authors.

\section{Author details}

'Department of Ophthalmology, University of California, San Francisco, 490 Illinois Street, San Francisco, CA 94158, USA. ${ }^{2}$ State Key Laboratory of Ophthalmology, Zhongshan Ophthalmic Center, Sun Yat-sen University, Guangzhou, China. ${ }^{3}$ Department of Ophthalmology, University of Pennsylvania, Philadelphia, PA, USA. ${ }^{4}$ Department of Ophthalmology, Stanford University, Stanford, CA, USA.

Received: 2 August 2021 Accepted: 23 November 2021

Published online: 20 December 2021

\section{References}

1. Stein JD. Serious adverse events after cataract surgery. Curr Opin Ophthalmol. 2012;23:219-25. https://doi.org/10.1097/ICU.0b013e3283524068.

2. Nemet AY, Assia El, Meyerstein D, Meyerstein N, Gedanken A, Topaz $M$. Protective effect of free-radical scavengers on corneal endothelial damage in phacoemulsification. J Cataract Refract Surg. 2007:33:310-5. https://doi.org/10.1016/j.jcrs.2006.10.031.

3. Masuda N, Maruyama A, Eguchi T, Hirakawa T, Murakami Y. Influence of microbubbles on free radical generation by ultrasound in aqueous solution: dependence of ultrasound frequency. J Phys Chem B. 2015;119:12887-93. https://doi.org/10.1021/acs.jpcb.5b05707.

4. Hayashi K, Hayashi H, Nakao F, Hayashi F. Risk factors for corneal endothelial injury during phacoemulsification. J Cataract Refract Surg. 1996;22:1079-84. https://doi.org/10.1016/S0886-3350(96)80121-0.
5. Hayashi K, Nakao F, Hayashi F. Corneal endothelial cell loss after phacoemulsification using nuclear cracking procedures. J Cataract Refract Surg. 1994;20:44-7. https://doi.org/10.1016/S0886-3350(13)80042-9.

6. Walkow T, Anders N, Klebe S. Endothelial cell loss after phacoemulsification: relation to preoperative and intraoperative parameters. J Cataract Refract Surg. 2000;26:727-32. https://doi.org/10.1016/S0886-3350(99) 00462-9.

7. Zetterström C, Laurell CG. Comparison of endothelial cell loss and phacoemulsification energy during endocapsular phacoemulsification surgery. J Cataract Refract Surg. 1995;21:55-8. https://doi.org/10.1016/ S0886-3350(13)80480-4

8. O'Brien PD, Fitzpatrick P, Kilmartin DJ, Beatty S. Risk factors for endothelial cell loss after phacoemulsification surgery by a junior resident. J Cataract Refract Surg. 2004;30:839-43. https://doi.org/10.1016/S0886-3350(03) 00648-5.

9. Chylack LT, Wolfe JK, Singer DM, Leske MC, Bullimore MA, Bailey IL, et al. The Lens opacities classification system III. The longitudinal study of cataract study group. Arch Ophthalmol (Chicago, III 1960). 1993(111):831-6. https://doi.org/10.1001/archopht.1993.01090060119035.

10. Bourne RRA, Minassian DC, Dart JKG, Rosen P, Kaushal S, Wingate N. Effect of cataract surgery on the corneal endothelium: modern phacoemulsification compared with extracapsular cataract surgery. Ophthalmology. 2004;111:679-85. https://doi.org/10.1016/j.ophtha.2003.07.015.

11. Pirazzoli G, D'Eliseo D, Ziosi M, Acciarri R. Effects of phacoemulsification time on the corneal endothelium using phacofracture and phaco chop techniques. J Cataract Refract Surg. 1996;22:967-9. https://doi.org/10. 1016/S0886-3350(96)80200-8.

12. Davison JA, Chylack LT. Clinical application of the lens opacities classification system III in the performance of phacoemulsification. J Cataract Refract Surg. 2003;29:138-45. https://doi.org/10.1016/s0886-3350(02) 01839-4.

13. Ursell PG, Spalton DJ, Tilling K. Relation between postoperative bloodaqueous barrier damage and LOCS III cataract gradings following routine phacoemulsification surgery. Br J Ophthalmol. 1997;81:544-7. https://doi. org/10.1136/bjo.81.7.544.

14. Storr-Paulsen A, Norregaard JC, Ahmed S, Storr-Paulsen T, Pedersen TH. Endothelial cell damage after cataract surgery: divide-and-conquer versus phaco-chop technique. J Cataract Refract Surg. 2008;34:996-1000. https://doi.org/10.1016/j.jcrs.2008.02.013.

15. Abell RG, Kerr NM, Vote BJ. Toward zero effective phacoemulsification time using femtosecond laser pretreatment. Ophthalmology. 2013;120:942-8. https://doi.org/10.1016/j.ophtha.2012.11.045.

\section{Publisher's Note}

Springer Nature remains neutral with regard to jurisdictional claims in published maps and institutional affiliations.
Ready to submit your research? Choose BMC and benefit from:

- fast, convenient online submission

- thorough peer review by experienced researchers in your field

- rapid publication on acceptance

- support for research data, including large and complex data types

- gold Open Access which fosters wider collaboration and increased citations

- maximum visibility for your research: over 100M website views per year

At BMC, research is always in progress.

Learn more biomedcentral.com/submissions 